\title{
2) Désaccord aux frontières et politique des visas : les relations entre Schengen et l'Union
}

\section{Elspeth Guild et Didier Bigo}

\section{(2) OpenEdition \\ 1 Journals}

\section{Édition électronique}

URL : http://journals.openedition.org/conflits/927

DOI : $10.4000 /$ conflits.927

ISSN : $1777-5345$

Éditeur :

CCLS - Centre d'études sur les conflits lilberté et sécurité, L'Harmattan

\section{Édition imprimée}

Date de publication : 1 mars 2003

Pagination : 38-70

ISBN : 2-7475-4868-8

ISSN : 1157-996X

\section{Référence électronique}

Elspeth Guild et Didier Bigo, «2) Désaccord aux frontières et politique des visas : les relations entre Schengen et l'Union », Cultures \& Conflits [En ligne], 49 | printemps 2003, mis en ligne le 29 septembre 2003, consulté le 30 mars 2021. URL : http://journals.openedition.org/conflits/927 ; DOI : https:// doi.org/10.4000/conflits.927

Ce document a été généré automatiquement le 30 mars 2021.

Creative Commons License 


\title{
2) Désaccord aux frontières et politique des visas : les relations entre Schengen et l'Union
}

\author{
Elspeth Guild et Didier Bigo
}

2) Désaccord aux frontières et politique des visas : les relations entre Schengen et l'Union

Avant de discuter des jeux politiques entre les divers acteurs que sont la Commission Européenne, les Etats de l'Union, les Etats membres de l'espace Schengen et les individus dans la relation entre eux qui se manifeste par l' obtention d'un visa dit Schengen, et dit uniforme, il est nécessaire de revenir sur l'économie des luttes entre les acteurs depuis 1985, afin d' éviter le récit habituel et ritualisé sur la soi-disant suppression des contrôles aux frontières intérieures, leur renforcement aux frontières extérieures et la création d'une identité européenne diverse mais homogène au sein d'un espace territorial bien défini. Un bilan historique et juridique un tant soit peu critique montre en effet les limites évidentes d' un tel discours.

Le traité d'Amsterdam est entré en vigueur le 1er mai 1999. Par le protocole intégrant l'acquis de Schengen dans le cadre de l'Union Européenne, l' expérience de près de quinze ans menée par certains Etats sous forme d'une Convention internationale et par des négociations purement intergouvernementales était terminée. Certains y ont vu la grande réconciliation entre les promoteurs de Schengen et la totalité des membres de l'Union. Mais, quel est le bilan de cette expérience? Différentes perspectives sont ouvertes et touchent directement la manière dont on envisage la question des visas, soit comme stratégie de police à distance, déterminante pour assurer la « sécurité » à l'intérieur de l' Union, soit comme un simple complément aux mesures de contrôles frontaliers.

Selon les pays, leurs traditions juridiques, l'acceptation des règles communes, les interprétations données nationalement sur le visa uniforme Schengen, selon la participation intensive ou non à la fourniture de données à l'intérieur du SIS, on aura des prises de position différentes qui tiennent à la trajectoire de ces pays, au moment 
où ils ont rejoint l'espace Schengen, aux conditions d'acceptation de cet espace comme espace de libre circulation ou comme espace de contrôle.

Historique des débats : la question des visas et le contrôle aux frontières

L'accord initial de Schengen, signé le 14 juin 1985, par l'Allemagne, la Belgique, la France, le Luxembourg et les Pays-Bas, a mis en place la structure nécessaire à la suppression des contrôles sur les personnes et les marchandises aux frontières entre les Etats signataires. Il a été complété par la Convention de Schengen de 1990 qui a réglé les détails de la suppression des contrôles aux frontières communes entre les Etats contractants à travers la thèse des mesures compensatoires et la distinction entre frontières intérieures et extérieures. Cette convention n'est finalement entrée en application que le 26 mars 1995 pour les contrôles sur les personnes. La Convention détermine de manière détaillée les dispositions relatives à la suppression des contrôles aux frontières communes aux Etats signataires, la mise en ouvre des contrôles aux frontières extérieures des Etats signataires, ainsi que les règles relatives à la détermination de l'Etat responsable de l'examen de la demande d'asile et à la coopération policière. Elle a permis en théorie d'abolir les contrôles aux frontières intérieures entre les Etats signataires et de créer une frontière extérieure unique où sont effectués les contrôles d'entrée dans l'espace Schengen selon des procédures identiques. Des règles communes en matière de visa, de droit d'asile et de contrôle aux frontières externes ont été adoptées ou doivent l'être afin de permettre à l'objectif affiché, la libre circulation des personnes au sein des pays signataires, de se mettre en place mais sans perturber pour autant la sécurité publique.

Cette rhétorique sur la naissance du "laboratoire Schengen ", sur la "coopération renforcée » en matière de libre circulation des Etats Schengen, sur leur volonté d'être plus pro-européen que les autres, est devenue si prégnante que les acteurs du champ n'osent s'en départir ou la remettre en cause officiellement. Elle a acquis la force d'un mythe ${ }^{1}$. Pourtant le « laboratoire » est plutôt de l'ordre du «bricolage » face à une crise soudaine puis à des préoccupations de sécurité et de peurs des migrations. Les acteurs $\mathrm{du}$ « laboratoire » que sont les cinq Etats du Benelux, la France, et l'Allemagne ne sont nullement pour la libre circulation prônée par la Commission. Les termes initiaux qu'ils utilisent sont ceux de mesures de sauvegarde face à la libre circulation, avant de les euphémiser ensuite en mesures compensatoires puis mesures d'accompagnement. Néanmoins cette euphémisation due à la plus grande publicité des accords en dit long sur l' esprit initial des acteurs policiers de Schengen et il n'est pas sûr qu'ils aient véritablement changé depuis. Quant à l'idée que ces acteurs seraient aussi plus proeuropéens que les autres, elle reste à démontrer quand on voit leurs résistances à la libre circulation des pays candidats comme nous venons de le signaler. Il faut donc rétablir brièvement une histoire moins "enchantée » de Schengen. On en trouvera un récit plus détaillé ailleurs ${ }^{2}$.

L'accord de Schengen est né de diverses pressions économiques, dont celle exercée par le secteur des transports afin de faire disparaître tous les obstacles au commerce entre Etats membres de l'Union européenne. La Commission européenne avait annoncé cet objectif dans son livre blanc sur l' achèvement du marché unique. Pourtant, il était très difficile, sur un plan politique, de parvenir à un accord sur le transfert vers la Communauté européenne de ces matières considérées comme des domaines sensibles relevant de la souveraineté nationale dans la mesure où la libre circulation des services induit la libre circulation des personnes. Le résultat de ces négociations politiques difficiles a été le maintien dans le domaine des négociations intergouvernementales de 
la circulation des ressortissants des pays tiers et de la coopération policière ${ }^{3}$. L'image du « laboratoire " Schengen est donc largement erronée dans la mesure où Schengen s'est constitué tout autant pour éviter que la Commission ne prenne en charge ce domaine que pour l'aider et pour anticiper les mesures soi-disant bloquées par le gouvernement britannique ${ }^{4}$. La Commission n'était d'ailleurs pas le seul adversaire. La Cour Européenne de Justice qui avait donné raison à la Commission contre les Etats membres dans son interprétation de la décision de 1984 sur sa capacité à coordonner les politiques migratoires, avait fabriqué des motifs très puissants de la part des ministères de l'Intérieur pour constituer un nouveau «club» leur permettant d'agir sans la Commission. D'ailleurs, l'accord de Schengen se voulait au départ confidentiel et ce sont les parlementaires de plusieurs pays - choqués par la publication discrète au journal officiel d'une mesure qui aurait dû passer devant eux - qui ont obligé les gouvernants à préparer la Convention de l'accord de Schengen en 1990. Si, a posteriori, on parle de coopération renforcée avant la lettre dans le cas Schengen, si l'on vante "l'acquis Schengen », l'esprit des premiers participants n'était nullement celui-là. Il fallait selon eux « sauvegarder » la sécurité menacée par les déclarations du livre blanc et de M. Adonino sur la suppression des douanes et l'ouverture des frontières, symbole d'une nouvelle citoyenneté européenne. Il fallait respecter la "souveraineté des Etats en l'organisant et non en la dissolvant $»^{5}$. Le cadre intergouvernemental était central. On pensait à l'époque marginaliser ainsi la Commission tout en étant efficace ${ }^{6}$. Concession néanmoins de dernier moment, il était prévu que les mesures prises dans le cadre de l'Union se substitueraient à celles de la Convention Schengen? .

En 1990, le discours officiel en France comme en Allemagne a été de présenter Schengen comme un point d'équilibre. Ni Europe passoire, ni Europe forteresse, Schengen visait selon ses promoteurs à concilier liberté et sécurité à l'échelle européenne. Il était donc logique que la nouvelle liberté de libre circulation soit, disaiton, encadrée et accompagnée par des mesures «compensatoires ». Ceci d'autant plus que l' llemagne - qui se voulait libérale - s'inquiétait de la porosité de sa frontière orientale et que certains cyniquement vantaient la «sûreté » de la période précédente ${ }^{8}$. Outre la gestion des contrôles aux frontières, il s' agissait d'en profiter pour moderniser les équipements, réduire un certain syndicalisme, et "améliorer la coordination entre les services de police, des douanes et de justice», en prenant les mesures nécessaires pour combattre notamment le terrorisme (via les accords de Trévi différents dans leur statut de la convention Schengen), la criminalité organisée ou simplement "sérieuse " et l'immigration illégale (au sein du groupe ad hocimmigration et de la Convention de Schengen). On verra ainsi une convention qui rappelle en un article la libre circulation mais vise dans tous les autres le renforcement de la coopération policière et judiciaire répressive, avec entre autres, l'instauration d'un droit de filature ou d'observation et de poursuite d'un pays à l'autre, le renforcement de la coopération judiciaire à travers un système d'extradition plus rapide et une meilleure transmission de l'exécution des jugements répressifs. Pour ce faire, un système d'information complexe était mis en place pour échanger des données concernant l'identité des personnes et la description des objets recherchés, le Système d'Information Schengen (SIS) et les SIRENE ${ }^{9}$. C'est ce que la presse retiendra le plus sans véritablement en comprendre la portée à l'époque et en n'y voyant qu'un fichier supplémentaire contre le crime.

France et Allemagne, mais aussi les pays du Benelux ont finalement, dans la même lignée de pensée des contrôles aux frontières intérieures, développé plus ou moins des moyens de contrôler autrement ces frontières intérieures avec, outre le SIS, la mise en 
place de centres de coopération policière et douanière (CCPD) $)^{10}$, avec $\mathrm{l}^{\prime}$ augmentation du pouvoir des douanes en matière de contrôles d'identité, avec la zone des vingt kilomètres et avec une augmentation constante des sanctions aux transporteurs, sanction se constatant à la frontière mais dissuadant ces derniers de faire voyager des personnes dont la validité des documents semble douteuse ${ }^{11}$.

Au cours des sept années qui suivirent (1990-1997), tous les autres Etats membres de l'Union européenne, à l'exception de l'Irlande et de la Grande-Bretagne, ont adhéré au système Schengen ainsi constitué et dont la mise en application s'est faite simplement en $1995^{12}$. Cela n'a pas débouché sur la suppression effective des contrôles aux frontières intérieures entre Etats membres, bien que cet objectif figurait, entre autres, au nombre des objectifs du marché unique, introduit, par l'Acte unique européen, à l'article 14 dans le traité $C E$. Les contrôles aux frontières intérieures ont été délocalisés en aval et rendus plus mobiles. Ils ont aussi en partie été privatisés. Ainsi, la technique des négociations intergouvernementales qui a été formellement reconnue, au sein de l'Union européenne, en 1993, par le biais de la création du troisième pilier réalisée par le Traité sur l'Union européenne a réussi son objectif principal: limiter les effets juridiques de la libre circulation, en particulier en ce qui concerne la libre circulation des étrangers. Finalement, la structure initiale de Maastricht a été modifiée et consolidée par le Traité d'Amsterdam de $1997^{13}$. Une partie de la Commission, celle travaillant sur le troisième pilier a été recrutée parmi les adversaires de la libre circulation et a joué un rôle clé pour faire admettre un rôle de la Commission mais un rôle de coordination des volontés des ministères de l'Intérieur et de la Justice, bien plus qu'un rôle de promoteur des valeurs de liberté. C'est à ce prix que l'acquis Schengen, comme on l'a désigné, a été communautarisée ${ }^{14}$.

Un des points communs de l'approche intergouvernementale, à l'égard aussi bien des ressortissants d'Etats tiers que de la coopération policière, avait été la mauvaise grâce des Etats à admettre qu'un contrôle judiciaire supranational soit exercé sur les questions d'interprétation et $d$ ' application des accords de Schengen ou du troisième pilier du Traité sur 1' Union européenne. La position des différents Etats membres à l'égard d'un contrôle judiciaire n'était en aucune façon monolithique. Comme Jean Rossetto l'a souligné, certains Etats membres avaient adopté une position très ferme en faveur d'un contrôle judiciaire supranational exercé par la Cour de Justice des Communautés Européennes, tandis que d'autres Etats membres y étaient opposés de manière absolument inflexible. Ces deux extrêmes sont habituellement incarnés par les Pays-Bas, favorables au contrôle judiciaire, et la Grande-Bretagne, fermement opposée à celui-ci ${ }^{15}$. En effet, l'opposition de la Grande-Bretagne à l'égard de l'harmonisation dans le domaine des contrôles aux frontières sur les ressortissants d'Etats tiers est devenue telle qu' elle refusa, en se fondant sur le droit souverain de tout Etat de maintenir des contrôles à ses frontières, de participer à l'expérience de Schengen ou de satisfaire aux objectifs de l'article 14 du Traité CE relatif à l' abolition des contrôles sur les personnes aux frontières internes à la Communauté ${ }^{16}$. Le gouvernement néerlandais, dont le Parlement a joué un rôle d'aiguillon important, a de son côté pris parti en faveur d'un contrôle judiciaire supranational sur le traitement des ressortissants d'Etats tiers et la coordination des différents dispositifs de contrôle. Le fait que ces matières soient en liaison avec la question des droits de l'Homme n'y est pas étranger. Les gouvernements sont donc souvent en lutte sur l'Europe qu'ils désirent et sur le fait de savoir si l'Union est plus ou moins autonome à l'égard des gouvernements nationaux, plus ou moins un centre de décision pouvant sanctionner 
des comportements des gouvernants. Les évolutions vers une Charte puis vers la Convention semblent aller à contrario de celles prises à Tampere, Laeken et Séville. La Cour de Justice va jouer un rôle. Le vieil adversaire ressurgit.

Par ailleurs, au cours des années 1980, un débat s'est ouvert sur la signification de la notion de « libre circulation des personnes » et l'abolition des contrôles aux frontières intérieures. Pour certains Etats membres, dont la France, la libre circulation ne devait s' appliquer qu'aux seuls citoyens européens ce qui impliquait de conserver les contrôles aux frontières intérieures pour distinguer citoyens européens et ressortissants des pays tiers. D'autres Etats membres, dont les Pays- Bas, au contraire, souhaitaient établir une libre circulation pour tous et par conséquent supprimer ces contrôles frontaliers. Bien que ces derniers, après un changement d'attitude momentané de l'Allemagne, aient triomphé, les visions s'opposent toujours. Qu'est-ce que la citoyenneté européenne? Est-elle réductible à la citoyenneté des Etats qui composent l'Union? Qu'en est-il de tous les étrangers vivant dans cet espace? L'obligation de déclaration pour tout ressortissant de pays tiers qui circule d'un pays à un autre n'a pas réglé la question. La déclaration d'entrée des étrangers aux frontières intérieures qui continuait théoriquement d'être obligatoire en France dans la mesure où son défaut vaut expulsion, est tombée en désuétude, vu les difficultés pratiques de la disposition qui suppose la pleine et entière collaboration des étrangers si on ne peut contrôler tout le monde afin de détecter qui est citoyen des pays membres et qui ne l'est pas. Mais on s'est bien gardé d'en faire un thème de débat car cela supposerait de revenir sur les catégories fondant l'espace de citoyenneté de l'Union. La désuétude de cette déclaration d'entrée est néanmoins symptomatique d'une vision particulière qui met l'accent sur la sécurité aux frontières mêmes et qui pose un principe de défiance par rapport aux étrangers alors que d' autres pays et d'autres acteurs - comme par exemple les ONG en faveur d'une citoyenneté européenne étendue aux étrangers y vivant -, mettent l'accent sur le droit intangible à la libre circulation sans contrôle. Cette différence de principe joue autant sur les conditions de mise en place des contrôles que sur ce que l'on attend en pratique des pays candidats dans le processus d'élargissement. Les enjeux identitaires de l'Union Européenne, de sa capacité à permettre à des étrangers d'y voyager, d'y séjourner et d'en repartir sans problème sont liés aux visions de l'étranger comme ami, ennemi ou simple quidam de passage envers qui indifférence et tolérance sont possibles et ne nécessite de sa part que respect des coutumes locales et des règles de droit sans nécessaire intégration. Le problème tient à ce que chaque Etat de l'Union a sa vision des étrangers et des étrangers dangereux, chaque Etat a ses propres peurs. Et à l'intérieur de chaque Etat le clivage (in)sécuritaire en vient à remplacer celui traditionnel entre la droite et la gauche. Il devient alors un enjeu électoral disproportionné avec les risques effectifs, car si l'on se souvient des peurs de voir lors des premiers élargissements les Grecs et les Portugais envahir la France et l' Allemagne, on ne peut que sourire, car les plaintes actuelles de la Commission et du Conseil sont liés à l'absence de mobilité intraeuropéenne ${ }^{17}$.

Sans doute les positions respectives des Etats tiennent elles à la présence ou non d'un populisme anti-étranger, à la structure du jeu politique et à l' histoire propre du pays depuis le début du vingtième siècle. Cela tient aussi à une certaine situation géographique. Concernant le «report " des contrôles aux frontières extérieures, la définition commune des conditions de franchissement de ces frontières, et la séparation dans les aérogares et les ports, entre les voyageurs voyageant au sein de 
Schengen et ceux provenant de l'extérieur de la zone, des débats importants ont aussi eu lieu. Certains pays membres ont voulu que le report se traduise par une quasi systématicité des contrôles aux frontières extérieures et que la surveillance soit renforcée. La France qui a l'avantage d'avoir très peu de frontières terrestres extérieures est parmi les pays les plus exigeants sur ce point. Elle a été le pays le plus critique sur les entrées de l'Italie puis de la Grèce et continue à vouloir un degré "fort » de sécurité aux frontières lorsque celles-ci seront de la responsabilité des pays candidats à l'Union. La France, par l'intermédiaire de ses parlementaires, a proposé à plusieurs reprises la mise en place d'une coordination entre administrations pour surveiller les frontières (officiers de liaison, harmonisation des instructions et de la formation données au personne) et l'envoi de groupes multinationaux sur les frontières à risques (la mise en place d'un corps multinational de garde-frontières est envisagée dès 1990 par Paul Masson, sénateur français). Les Pays-Bas - qui ont une position différente de la France sur les frontières intérieures et sur le rôle de la Cour de Justice sont en revanche partisans eux aussi d'un fort contrôle aux frontières extérieures en raison même de l'absence de contrôle qu'ils veulent appliquer ensuite. L'Allemagne, qui durant la phase de négociation avant la chute du mur était très rigide, a changé de position après la réunification. Elle a voulu une politique plus souple pour dynamiser les échanges transfrontières entre l'Allemagne, l'Autriche, la Pologne et la Hongrie. Selon les positions des ministères, selon les Länderet les alternances politiques, la situation varie. Dans les déclarations de principes tournées vers les partenaires de Schengen, l' Allemagne clame qu'elle assure des contrôles efficaces. En Pologne, elle insiste sur la souplesse des arrangements. En pratique, elle cherche une autre gestion politique des flux et elle est sans doute le pays le plus actif pour mettre en place la politique de police à distance, via les consulats. Beaucoup d'experts allemands sont partis aux Etats-Unis pour regarder leur gestion avec le Canada et le Mexique. L'Allemagne, à l'heure actuelle, cherche à la fois à obliger la Pologne à renforcer les contrôles à sa frontière orientale sans dégarnir la frontière occidentale, et surtout elle insiste pour l'harmonisation des conditions d'entrée et de visas pour les courts séjours. Etant donné la longueur de sa frontière terrestre et l' attirance des étrangers pour son économie, elle veut «faire partager le fardeau » en matière de flux de personnes et non simplement en matière d'asile. Elle prône les accords de réadmission et la coopération consulaire.

Les pays du Nord et du Sud de l'Europe ont des positions moins crispées sur la gestion des frontières. Les pays du Nord ont depuis longtemps un accord de libre circulation entre eux qui oblige Islande et Norvège (bien que non membres de l'Union Européenne) à appliquer l'acquis Schengen. Ils mettent l' accent avant tout sur la collaboration policière et douanière transfrontalière à l'instar de la Finlande avec la Russie. Italie, Grèce, Espagne ne veulent pas être les cibles des attaques de la France et de l' Allemagne à propos des migrations clandestines. Ces pays sont prêts à prendre des mesures symboliques pour la gestion des opinions publiques des pays du noyau Schengen initial, mais ils ne sont pas disposés à lancer des programmes très coûteux et sans doute peu efficaces de contrôle de leurs côtes. La Grèce en particulier ne peut gérer l'ensemble de ses îles et considère que le tourisme passe avant les préoccupations de sécurité en matière migratoire des autres pays. Elle estime qu'une gestion trop rigide serait néfaste économiquement et que les peurs migratoires d'invasion ne se justifient nullement. Dès lors, ces pays sont aussi, comme l'Allemagne, en faveur d'une gestion à distance des frontières et de la libre circulation. Il leur semble que la politique 
des visas est une politique plus cohérente que celle des contrôles systématiques aux frontières extérieures. La création d'un corps de garde frontières travaillant en commun au niveau de 1 'Union Européenne est alors plus de l'ordre symbolique que pratique. C'est ce qui explique l'incompatibilité des positions de la Commission en faveur d 'un corps unique et l'étude de faisabilité italienne qui ne veut qu'un réseau de garde-frontières nationaux ${ }^{18}$.

Tous les gouvernements ont justement tendance à se retrouver, malgré leurs différences sur la gestion des contrôles aux frontières intérieures et extérieures, lorsqu'il s'agit de faire porter la responsabilité sur les acteurs privés que sont les transporteurs et ils s'interrogent peu sur la délégation d'activités régaliennes à ces entreprises, même lorsque certains hommes politiques se disent très sourcilleux sur la souveraineté. Le renforcement des sanctions des transporteurs pose de graves problèmes que nous ne développerons pas ici ${ }^{19}$. Il met en question la possibilité de fuir son pays en cas de danger et remet le destin de certaines personnes dans les mains d'agents privés non formés à prendre des décisions dans des domaines aussi graves. Plus généralement, la délégation des activités de contrôle tend à être permanente, même en cas de dysfonctionnement, ce qui pose aussi des problèmes de sécurité. Cette forme de gestion à distance espérant bloquer les voyages et dissuader les partants est une politique qui n'a guère de chance de succès sur le moyen et long terme. Les compagnies, soit ne seront pas sanctionnées malgré la loi, soit protesteront en groupe à l'échelle européenne. Les syndicats de pilotes de ligne de diverses compagnies ont déjà commencé à se réunir pour coordonner leurs actions. Malgré tout, la directive qui fait suite à la proposition de la présidence française d'harmoniser les sanctions contre les transporteurs (dans le cas où ils n'acceptent pas la responsabilité de contrôler les personnes soumises à une obligation de visa) a été présentée comme s'il s' agissait d'une solution face au trafic de personnes en exploitant l'émotion de Douvres ${ }^{20}$. Or, on peut en douter quand on voit les réactions des entreprises de transport ${ }^{21}$.

On le voit, les divergences d'appréciation de la Convention Schengen à l' égard des contrôles aux frontières sont extrêmement importantes d'un pays à l'autre, dès que l'on regarde les pratiques et non les déclarations $d$ ' intention des gouvernants. Chaque pays mène sa politique à l'égard de la gestion des contrôles aux frontières intérieures et extérieures en fonction de ce qu'il définit comme ses intérêts propres et il n'est pas légitime de parler d'un régime international de normes Schengen comme le font de plus en plus d'études internationalistes ${ }^{22}$. Dire cela ne signifie pas pour autant faire retour à une lecture neo-réaliste car les gouvernements doivent transiger au sein d'arènes spécifiques et ne peuvent continuer à jouer « cavalier seul » comme l'a montré finalement l'échec de la position d'isolement britannique et son obligation d'intégrer par petits pas les normes Schengen ${ }^{23}$. Il faut analyser l'Europe comme un champ de contraintes et d'opportunités fortement segmenté selon des logiques professionnelles et ministérielles. La constitution d'un champ européen de la sécurité ne suit pas une hypothétique intégration politique ou économique. Il se constitue avec des acteurs différents provenant des champs politiques nationaux mais ayant une spécialisation dans le domaine de la sécuritée ${ }^{24}$.

Ce domaine est lui-même toujours en discussion et ses frontières ont évolué. Les relations sécurité extérieure, intérieure et migration ont varié au cours du temps. Leur institutionnalisation en termes de pilier n'a pas empêché les recoupements et les luttes d'influence. Les règles du jeu ont été modifiées et de nouveaux acteurs en ont profité. Cela permet par exemple de comprendre l'évolution de rapports de forces qui ont 
évolué en faveur de la Commission, si l'on prend une perspective de plus de dix ans. Exclue du jeu au milieu des années 1980, avec Schengen et Trevi, elle a réussi d'abord à prendre un rôle modeste mais effectif avec la constitution du troisième pilier de Maastricht et ensuite via la restructuration de ce dernier dans le traité d'Amsterdam et la naissance d'un titre IV consacré au franchissement des frontières aux visas et à l'asile, elle a pu peser d'un poids plus grand. Néanmoins, l'enjeu central reste que le système Schengen est bien plus une multilatéralisation d'accords bilatéraux qu'une uniformisation des accords.

Or la logique poursuivie par la Commission est plus ambitieuse. Elle cherche à faire du territoire européen non quinze - ou treize dans le cas de Schengen - territoires coordonnés mais un seul et unique territoire. Certains Etats ne veulent pas aller vers l'unification d'un territoire européen comme le montrent les résistances au passage à des procédures créant un échelon supérieur au niveau de l'Union. Le parallèle avec ce qui se fait en matière de justice est éclairant. Certains veulent simplement la reconnaissance mutuelle des décisions de justice, et la création d'un réseau de magistrats nationaux, alors que d'autres veulent s'engager vers la création d'un procureur européen compétent pour les fraudes européennes et vers un corpus jurisuniformisant les règles et le territoire applicable. Avec l'intégration de l'acquis Schengen au sein du traité CE cette opposition entre les deux logiques est brouillée. L'acquis peut alors être autant un frein à l'harmonisation qu'une incitation.

Que la Commission ait plus de responsabilités ne signifie en rien qu'elle aurait une optique moins sécuritaire que les gouvernements comme certaines ONG ont tendance à le croire $^{25}$. Dans ce domaine, la Commission a donné des gages aux Etats les plus puissants et ceux qui ont préparé les textes de la Commission avant Amsterdam et Tampere ont parfois déplacé le centre de gravité en faveur des positions les plus "dures ", comme en matière d'interprétation de la Convention de Genève sur le droit d'asile. En revanche, les acteurs de la Commission qui travaillaient auparavant dans la division marché intérieur ont des positions différentes sur l'immigration et ils jouent maintenant un rôle dans la division de Vitorino ${ }^{26}$.

L'intégration de l'acquis Schengen dans le traité de l'UE : la Commission dans le jeu des Etats

La situation actuelle est donc celle d'une production normative où la Commission joue un rôle non négligeable mais où les Etats restent maitres pour le moment de l'application pratique des règles. Le Traité d'Amsterdam, entré en vigueur le 1er mai 1999, comprend maintenant un protocole relatif à Schengen qui introduit en fait dans le Traité $\mathrm{CE}^{27}$ (ci-après TCE) l'accord de Schengen de 1985, la Convention de Schengen de 1990 ainsi que toutes les décisions prises par le Comité exécutif dans ce cadre dans la mesure où elles concernent des questions de frontières et de ressortissants d'Etats tiers. Ce même protocole introduit dans le troisième pilier du Traité sur l'Union européenne les dispositions de Schengen relatives à la coopération policière, à l'entraide judiciaire en matière pénale et au SIS. La Grande-Bretagne, l'Irlande et le Danemark - qui pour ce dernier fait partie néanmoins de Schengen - ont tous trois négocié des protocoles qui leur permettent de demeurer pour l'instant en dehors de la nouvelle réglementation communautaire en matière de frontières et de ressortissants d'Etats tiers. Il appartiendra au Royaume-Uni et à l'Irlande de décider au cas par cas s'ils souhaitent ou non participer au nouveau système mis en place. 
L'intégration de l'acquis Schengen dans le cadre de l'Union européenne change tout de même en partie les procédures de décision. Tout d'abord, comme le prévoyait le traité d'Amsterdam, le Conseil s'est substitué au Comité exécutif créé par les accords de Schengen. Le 1er mai 1999, le Conseil a fixé les modalités de l'intégration du secrétariat de Schengen au secrétariat général du Conseil, notamment celles relatives au personnel employé par le secrétariat de Schengen ${ }^{28}$. Par la suite, de nouveaux groupes de travail ont été créés pour aider le Conseil à gérer la situation. Ensuite, le Conseil, assisté du personnel de la Commission, avait été chargé d'un très long travail de répartition des décisions prises dans le cadre du comité exécutif Schengen pour savoir si elles avaient valeur contraignante ou non et si elles relevaient du troisième pilier d'Amsterdam ou $\mathrm{du}$ titre IV du premier pilier. Il en résulte un très long document qui a sélectionné parmi les dispositions et les mesures prises par les Etats signataires de ces accords intergouvernementaux, celles qui constituaient un véritable acquis, c' est-à-dire un ensemble d'actes à conserver pour permettre de poursuivre la coopération entreprise. Une liste des éléments composant l'acquis a été adoptée le 20 mai 1999 ainsi que la définition pour chacun d'entre eux de la base juridique correspondante dans les traités européens (traité CE ou traité sur l'UE) ${ }^{29}$. Ces éléments sont publiés au Journal officiel sauf ceux considérés comme confidentiels. Cette publication est d'autant plus importante qu'ils font partie des règles juridiques que les pays candidats à l'adhésion doivent reprendre dans leur législation nationale. Considéré comme le premier exemple concret de "coopération renforcée » entre treize Etats membres, l'espace Schengen rejoint aussi le cadre juridique et institutionnel de l'UE au sein duquel il bénéficie d'un contrôle parlementaire et juridictionnel (Cour de justice et/ou juridictions nationales selon les domaines mais avec des exceptions notables). Au sein de la Commission, c'est l'unité JAI de M. Vitorino qui est en charge de la vérification des pratiques consulaires de délivrance des visas et de la vérification et adaptation des deux listes des pays dont les ressortissants sont soumis ou non à visa.

La Commission a choisi l'outil du règlement et non celui de la directive pour tenir des délais courts. Ce choix du recours à un règlement plutôt qu'à une directive s'explique aussi par diverses raisons techniques et politiques : via le règlement, la fixation de listes de pays dont les ressortissants sont soumis à l'obligation de visa ou en sont exemptés ne laisse pas de marge de manouvre aux Etats membres pour «transposer » ces listes. En outre, un retard de transposition de l'un ou l'autre Etat membre entraînerait de multiples difficultés pratiques, dont certaines seraient de nature à remettre en cause le fonctionnement du régime des visas résultant de l'acquis Schengen intégré dans l'Union si on avait choisi une directive. Par ailleurs, les Etats sont davantage liés par ce système de deux listes exhaustives qu'auparavant, même s'ils ont tenté, via les décisions liées à 1 'annexe $5 \mathrm{~b}$, de retrouver des marges de manouvre ${ }^{30}$.

La Commission a donc commencé son travail normatif par une proposition de règlement supprimant la possibilité pour les Etats d'ajouter à volonté des pays à la liste des Etats dont les ressortissants sont soumis à visa et a essayé "d'harmoniser " au sens d'une nécessaire collaboration entre les Etats et d'un accord entre eux « une » liste. Officiellement c'est une réussite puisqu'en deux ans on est parvenu à un accord.

L'article 62 du traité CE disposait que toutes les mesures qu'il vise (en particulier la liste des pays tiers dont les ressortissants sont soumis à visa et de ceux qui en sont exemptés) doivent être arrêtées par le Conseil dans les cinq ans qui suivent l'entrée en vigueur du traité d'Amsterdam. Le plan d'action du Conseil et de la Commission du 3 
décembre $1998^{31}$ approuvé par le Conseil européen de Vienne des 11 et 12 décembre 1998, avait "resserré » par rapport au traité le calendrier de certaines des mesures à arrêter dans le cadre du titre IV du traité CE. C'est ainsi que l'élaboration d'un règlement relatif aux pays tiers dont les ressortissants sont soumis à visa et de ceux qui sont exemptés de cette obligation figure dans le plan d'action parmi les «mesures à terme de deux ans ». Le Conseil européen de Tampere des 15 et 16 octobre 1999 avait rappelé à cet égard la nécessité de la mise en ouvre intégrale et immédiate du traité d'Amsterdam, sur la base du plan d' action de Vienne. La décision devait donc être prise avant décembre 2000 .

En définitive, le nouveau règlement 539 a été adopté le 15 mars 2001 par le Conseil ${ }^{32}$ et est entré en vigueur le 10 avril 2001, soit vingt jours après sa publication au Journal Officiel des Communautés européennes. Dans son exposé des motifs pour la proposition de Règlement fixant la liste des pays tiers dont les ressortissants sont soumis a l'obligation de visa pour franchir les frontières extérieures et la liste des pays dont les ressortissants sont exemptés de cette obligation, la Commission explique sa position en ces termes :

"Le traité d'Amsterdam constitue une avancée remarquable de l' intégration européenne dans le domaine de la politique des visas par rapport au traité de Maastricht. Ce dernier en introduisant l'article $100 \mathrm{C}$ dans le traité CE, avait communautarisé seulement deux aspects de la politique des visas (d'une part la détermination des pays tiers dont les ressortissants doivent être munis d'un visa lors du franchissement des frontières extérieures des Etats membres et d'autre part l'instauration d'un modèle type de visa). Le traité d'Amsterdam a par contre communautarisé tous les autres aspects de la politique des visas en les intégrant dans le nouveau titre IV du traité CE 'Visas, asile, immigration et autres politiques liées à la libre circulation des personnes' qui vise la création d'un espace de liberté, de sécurité et de justice. Simultanément, un protocole annexé au traité d'Amsterdam a intégré l'acquis de Schengen dans l'Union, ce qui comprend toute l'harmonisation en matière de visas à laquelle les Etats Schengen étaient parvenus " $^{33}$.

En effet, parmi les mesures mentionnées à l'article 62, point 2) b du traité CE sous l'intitulé "les règles relatives aux visas pour les séjours prévus d'une durée maximale de trois mois» figure explicitement "la liste des pays tiers dont les ressortissants sont soumis à l' obligation de visa pour franchir les frontières extérieures et de ceux dont les ressortissants sont exemptés de cette obligation ». La proposition de règlement 5392001 répond ainsi à l'article 62, point 2) b et comporte en annexe deux listes exhaustives des pays tiers. Un pays tiers figure obligatoirement ou bien dans l'annexe 1 (obligation de visa), ou bien dans 1 'annexe 2 (exemption de visa) ${ }^{34}$. La possibilité d'une détermination unilatérale par un Etat membre du régime de visa applicable semble ainsi exclue à l'égard de tous les pays tiers sans exception.

Mais ce discours officiel sur l'harmonisation ne saurait faire oublier que se maintiennent des différences fondamentales concernant le territoire d' application, les types de visas, le retour en force des Etats via des annexes confidentielles, un écartèlement $\mathrm{du}$ droit européen et une idéologie discutable dans laquelle harmonisation rime avec politique de contrôle, politique policière à distance et non libre circulation.

L'écartèlement du droit : résultat des antagonismes

L'écartèlement du droit est souvent mal apprécié par les non spécialistes qui n'en mesurent pas les effets et qui ne voient pas en quoi ceci met à mal les principes du droit européen. Mais il existe actuellement en fait deux sources de droit: le droit 
communautaire antérieur et le droit dérivé de l' acquis Schengen qui apparaît comme une insertion de l'exceptionalisme au sein d'un droit général.

Le droit communautaire

Sur la base de l'article $100 \mathrm{C}$, le Conseil avait arrêté un règlement - le règlement (CE) $n^{\circ} 2317 / 95$ [1] du 25 septembre $1995^{35}$ - qui, après son annulation par arrêt de la Cour de justice du 10 juin 1997, a été suivi par le règlement (CE) n ${ }^{\circ}$ 574/99 [2] ${ }^{36}$ déterminant les pays tiers dont les ressortissants devaient être munis d'un visa lors du franchissement des frontières extérieures des Etats membres. Ce règlement (CE) $n^{\circ} 574 / 99$, tout comme le règlement précédent (CE) n 2317/95, comportait uniquement la liste commune des pays tiers dont les ressortissants sont soumis à l'obligation de visa. Pour les pays tiers ne figurant par sur la liste commune, les Etats membres restaient libres de soumettre leurs ressortissants à l'obligation de visa ou de les en exempter. Une pratique différentiée s'était manifestée à 1 'époque, souvent liée aux traditions coloniales et aux liens culturels et linguistiques. Cet ensemble a été modifié partiellement par le règlement $n^{\circ} 539$ 2001, mais partiellement seulement.

Pour faire passer le projet, la Commission a en effet indiqué clairement que ce règlement ne couvre pas l'ensemble des questions, mais est spécifique. "Le règlement proposé, fondé sur l'article 62, point 2 b) i, ne peut pas :

- s'appliquer à des instruments qui ne relèvent pas de cette base juridique comme le visa de long séjour ou le visa de transit aéroportuaire ;

- fixer les modalités et conditions de délivrance des visas, lesquelles relèvent de l'article 62 , point 2 b) ii ;

- déterminer les conditions de circulation intracommunautaire des porteurs de visas ou des personnes exemptées de visa car cela sera fixé par un instrument fondé sur l'article 62 , point 3 ;

- comporter des dispositions qui entrent dans le domaine de la gestion du franchissement des frontières extérieures (par exemple : non admission à la frontière ou admission exceptionnelle à titre humanitaire ; délivrance de visas à la frontière).

Le règlement n'affecte pas la possibilité que des mesures exceptionnelles puissent être prises dans des cas particuliers. Il y a lieu de se reporter à cet égard à l'article 64, point 2, du traité CE qui prévoit la durée et les conditions permettant de telles mesures («situation d'urgence caractérisée par un afflux soudain de ressortissants de pays tiers ») et fixe la procédure à appliquer.

Le règlement n'affecte pas diverses dispositions de l'acquis Schengen intégré qui concernent les visas mais qui ne relèvent pas du champ $\mathrm{d}$ ' application du règlement (par exemple : la reconnaissance mutuelle des visas, l'équivalence entre titre de séjour et visa)».

On le voit la stratégie de la Commission a été marquée par la prudence. Les Etats veulent toujours pouvoir utiliser des clauses d'exception suffisamment générales et floues pour ne pas être tenus de rendre des comptes à la Commission. Mais grâce à cela le principe du rôle central de la Commission a été définitivement acquis. 
En revanche, la Commission a été bloquée dans sa volonté de réforme et $\mathrm{d}^{\prime}$ harmonisation par la création d'un soi-disant «acquis Schengen » sur lequel elle ne pouvait revenir frontalement et sur lequel le parlement européen n'a pas pu se prononcer sur le fond. Elle n'a pas pu obliger les Etats à aller vers un visa unique et le visa uniforme Schengen est en fait bien plus de l'ordre de la reconnaissance plus ou moins mutuelle de divers visas dont seule la forme est «harmonisée » pour des soucis de technique sécuritaire.

Le droit dérivé de Schengen

L'acquis Schengen et son intégration crée toujours des effets importants comme par exemple la quasi-paralysie de l'Autorité de contrôle commune des données vérifiant les inscriptions au SIS et le blocage des discussions de fond sur les raisons d'inscription d'un pays sur la liste des Etats dont les ressortissants sont soumis à visa. En effet, rappelons-le, dans le cadre de Schengen, certains des Etats membres avaient développé une coopération dite "plus étroite" dans le domaine des visas, c'est-à-dire une politique de contrôle et de police à distance plus sévère que celle des douze. La licéité d'une telle coopération au regard du droit communautaire avait été confirmée par les règlements $(\mathrm{CE}) \mathrm{n}^{\circ} 2317 / 95$ et 574/99 qui précisaient qu'ils ne faisaient pas obstacle à une harmonisation plus poussée entre les Etats membres, dont la portée irait au-delà de la liste commune. Mais, cette idée que l'harmonisation consistait par définition à ajouter plus de pays dans la liste «noire » a joué idéologiquement un rôle central en remplaçant l'idée d'une libre circulation mondiale comme objectif final par celle d'un contrôle de plus en plus répressif des flux de personnes voyageant pour des motifs touristiques, migratoires ou même d'asile. Les Etats Schengen en développant cette harmonisation plus poussée vis-à-vis des pays tiers ne figurant pas sur la liste des règlements $(\mathrm{CE}) \mathrm{n}^{\circ} 2317 / 95$ et 574/99 ont, en fait, avant tout mis en commun leurs peurs respectives.

L'harmonisation dans ce sens particulier que le terme a pris, s'est faite progressivement et son état final, au moment de l'intégration de Schengen dans le cadre de l'Union européenne, était la suivante :

une liste de 32 pays tiers qui ne figuraient pas sur la liste du règlement (CE) n 574/99 et dont les ressortissants étaient soumis à l'obligation de visa par tous les Etats Schengen ; une liste de 44 pays tiers dont les ressortissants étaient exemptés de visa par tous les Etats Schengen (il n'y avait pas d'équivalent dans le cadre du règlement (CE) n 574/99 qui ne comportait pas une telle liste);

Il est à noter que pour un seul pays tiers les Etats Schengen n'étaient pas parvenus à une position harmonisée, la Colombie. Le règlement 5392001 a réglé cette situation en faisant figurer la Colombie sur la liste des pays dont les ressortissants sont soumis à visa $^{37}$. Mais la Commission n'a pas discuté les raisons de l' inscription sur la liste, et encore moins le nombre impressionnant d'Etats dont les ressortissants sont soumis à visa, elle les a considéré comme un " acquis ».

Cet acquis Schengen fait donc maintenant, depuis l'entrée en vigueur du traité d'Amsterdam, partie intégrante du droit communautaire et continue à s 'appliquer entre les Etats Schengen mais toujours pas au Royaume-Uni et à l' Irlande. Le territoire Schengen est toujours différent du territoire de l' Union.

L'écartèlement de l'espace territorial malgré un cadre qui se voulait unifié 
Même après Amsterdam, l'espace Schengen qui comprend donc treize Etats membres plus deux membres associés (l'Islande et la Norvège) mais pas le Royaume-Uni et l'Irlande ${ }^{38}$, ne coïncide pas géographiquement exactement avec les frontières de l'Union.

Tout d'abord, il aurait été souhaitable que les micro-Etats (Liechtenstein, Monaco, Andorre) signent les accords ou des protocoles concernant des aspects de la Convention en matière de libre circulation et de coopération policière comme l'a fait la Suisse, par exemple, mais ce n'est pas le cas.

Ensuite, la partition organisée dans l'espace entre les métropoles et leurs possessions hors de l'espace européen - que l'on pense aux Antilles néerlandaises ou aux DOM-TOM français -, conduit à faire une différence à $\mathrm{l}^{\prime}$ intérieur des citoyens français ou néerlandais selon leur lieu de résidence. En France, Pierre Mazeaud avait été à l'initiative d'une demande des parlementaires sur l'inconstitutionnalité des accords de Schengen sur cette base, mais le conseil constitutionnel avait refusé l'argument et par la suite il avait été permis, par dérogation de soumettre à visa les personnes résidant dans les DOM-TOM ${ }^{39}$. Seulement, des ONG et des avocats ont fait savoir depuis que certains de leurs clients nationaux français avaient été enregistrés dans le SIS alors que ceci est formellement interdit sur la base de l'article 96 qui concerne les étrangers non communautaires indésirables. Des plaintes ont été déposées devant l'autorité commune de contrôle des données mais dans les quatrième et cinquième rapports qu'elle a publiés, si elle a fait état de question d'identités usurpées, elle n'a pas évoqué ces cas et on ne sait si elle a donné suite aux plaintes, exigé et obtenu la suppression de ces données.

Enfin, le fait que le Royaume-Uni et l'Irlande, malgré l'apaisement de la situation en Irlande du Nord, aient maintenu leur refus d'entrer dans l' espace Schengen a des conséquences graves. Cette "géométrie inédite» pour reprendre l'expression de Wenceslaw de Lobkowicz a pour effet de casser la naturalité de l'adéquation entre frontière extérieure de l'Union et visa pour entrer dans l'espace de l'Union puisqu'il y a toujours une procédure différente de visa pour le Royaume-Uni et l'Irlande ${ }^{40}$. Le visa dit uniforme ne l'est que dans l'espace Schengen. Cela n'en simplifie pas la gestion concrète et multiplie les ambiguïtés pour les étrangers refoulés par le Royaume-Uni vers un pays Schengen comme la Belgique ou la France. Le phénomène Sangatte, qui continue bien après la fermeture du camp ouvert par la Croix-Rouge pour les personnes en difficulté cherchant à traverser la Manche, est lié à ce problème structurel. Il n'aurait pas eu de raison d'exister s'il y avait eu unité territoriale de l'espace européen des visas. Mais là aussi toutes les polémiques autour de Sangatte entre les gouvernements français et britannique ont d'un commun accord évité d'aborder ce thème. Seules certaines ONG ont rappelé ce point. Il faut d'ailleurs ajouter qu'en vertu du protocole Schengen, le Royaume-Uni et l'Irlande peuvent à tout moment demander à participer à tout ou partie de l'acquis Schengen. La demande que le Royaume-Uni a introduite à cet égard, par lettre du 20 mai, du 9 juillet et du 6 octobre 1999 au Président du Conseil, porte sur une partie de l' acquis concernant le SIS mais pas sur des matières concernant les visas ${ }^{41}$. Ceci donne une position exorbitante au RoyaumeUni (et à l'Irlande) qui peuvent choisir à la carte ce qui correspond à leurs intérêts alors que les anciens membres Schengen, tout comme la Norvège et l' Islande et tout comme les pays candidats à l'entrée sont obligés de tout accepter ou de tout refuser. Le 
discours permanent sur l'harmonisation vise en fait à masquer ces différences cruciales.

Le Royaume-Uni a certes demandé en mars 1999 à participer à certains aspects de la coopération basée sur Schengen: la coopération policière et judiciaire en matière pénale, la lutte contre les stupéfiants et le système d'information (SIS). La Commission a formulé un avis favorable à cette demande le 21 juillet 1999, et malgré les réserves de l'Espagne qui voulait régler définitivement la question de Gibraltar avant l'entrée du Royaume-Uni au sein de la coopération policière Schengen, le Royaume-Uni est partie prenante au SIS - sauf article 96. En revanche, il ne participe pas à la libre circulation des personnes et veut continuer à assurer les contrôles des personnes à ses frontières. $C^{\prime}$ 'est en grande partie cette non coïncidence qui oblige dès lors les acteurs de Schengen à faire pression sur tous leurs voisins immédiats pour qu'ils adoptent les mêmes règles qu'eux et résolvent chez eux ce que la négociation interne n'a pu faire.

Le SIS comme instrument de gestion du crime et des étrangers aux frontières : une fausse harmonisation

S'il y a divergence dans les pratiques de contrôle aux frontières et si l' intégration de Schengen au sein de l'Union n'est pas aussi limpide qu'il y paraît, est-ce que ces divergences ou hétérogénéités sont relativisées par 1 'existence d'un seul et même outil auquel participeraient tous les Etats, à savoir le Système d'information Schengen (SIS) ? $C^{\prime}$ est ce que pensent certains de nos interlocuteurs qui en font la pierre angulaire de tout le dispositif.

Les hétérogénéités que nous avons mises en évidence seraient réelles mais marginales. Toute l'harmonisation passerait par le SIS qui jouerait un véritable rôle régulateur et serait l'outil approprié à la politique de lutte contre l'immigration illégale et la criminalité transfrontière.

Pourtant, là aussi, derrière l'apparente homogénéité du SIS, il y a, semble-t-il, autant de disparités dans l'entrée et la gestion des données d' un pays à l'autre qu'il y en a dans les procédures de contrôle aux frontières intérieures et extérieures et dans la politique dite harmonisée des visas.

Au cour du dispositif Schengen, un réseau automatisé a été élaboré, non sans difficultés techniques, pour permettre en théorie à tous les postes de police et les agents consulaires des Etats ayant adhéré à l'espace Schengen de disposer de données sur les personnes signalées, les objets ou véhicules recherchés.

Les Etats membres alimentent le SIS grâce à des réseaux nationaux (N-SIS) connectés à un système central (C-SIS) situé en France, à Strasbourg ${ }^{42}$. De plus, cette construction informatique qui permet une interrogation au fil de l'eau, est complétée par un réseau nommé SIRENE (supplément d'information requis à l' entrée nationale), composé de représentants de la police, de la gendarmerie, des douanes et aussi de la justice, lorsqu'il est nécessaire d'avoir des indications supplémentaires sur la conduite à tenir ou lorsqu'il faut transmettre des pièces ${ }^{43}$.

Le SIS est considéré comme l'élément central de l'espace Schengen et il est évident que son incorporation au sein de l'Union européenne ne devait causer aucun risque de rupture dans son fonctionnement. Le Conseil a été particulièrement attentif sur ce point. Aussi, différentes décisions d'ordre pratique ont-elles été prises afin de permettre au secrétaire général du Conseil de gérer certains aspects budgétaires du développement du SIS ${ }^{44}$. En effet, étant donné les contraintes contradictoires de sécurité et de rapidité d'accès aux données, le premier SIS est techniquement dans 
l'incapacité de gérer rapidement l'accès aux données pour treize pays. Un second SIS dit SIS2 a été décidé mais fin 2001, c'était encore un SIS1 amélioré qui fonctionnait ${ }^{45}$.

En 1999, les Etats membres ont envisagé de ne pas prolonger le contrat relatif au réseau SIRENE, celui-ci aurait dû s'achever le 23 août 2001. Mais à la suite des événements du 11 septembre, ils ont changé d'avis. Dès lors le problème a été la base légale du SIRENE. A terme, le SIRENE devrait se transformer en "système d'information européen" intégrant également des données sur l'immigration et sur toutes les personnes à qui un visa a été donné et dont le visa a expiré dans le but de savoir s'ils ont quitté le territoire européen. Dans ce cadre, une proposition du 5 et 6 juin 2003 demande que soit créée une base de donnée biométrique contenant des photographies numériques et des empreintes digitales au sein du SIS2.

Nous ne décrirons pas ici en détail le fonctionnement du SIS et du SIS2 pas plus que du projet de "Système d'Information Visa" (SIV ou VIS) qui fera l'objet d'un article spécifique lorsque les informations sur sa possible entrée en vigueur seront plus précises ${ }^{46}$. Il suffit pour notre sujet sur les visas de rappeler que les rubriques du SIS reprennent les articles de la Convention de Schengen (article 95 à 100) et que, normalement, chaque pays possédant la même passerelle et une compatibilité entre les données de son logiciel national et celui des autres, il devrait en résulter une harmonisation. On peut en effet comparer les données entrées par chaque pays sous une même rubrique et c'est d'ailleurs ainsi qu'elles sont présentées. Est-ce à dire que l'ensemble des pays participent à la fourniture des données disponibles dans le SIS ? Si les procédures de consultation sont relativement bien harmonisées d'un pays à l'autre, et si de plus en plus de consultations nationales font appel en parallèle au SIS par une lecture en double écran, il n'empêche que chaque pays a son interprétation des données qu'il doit inscrire dans le SIS.

Fin 2002 le SIS avait 11 millions d'entrées. 1,2 million concernait les personnes. 8,6 millions concernaient des documents ou objets perdus ou volés, le reste 1,1 million était essentiellement des véhicules. Les signalement français présents au SIS était de 3,1 millions dont 2680000 pour des documents, billets de banque ou armes perdues ou volées, 263000 véhicules volés, et 147000 personnes signalées dont 52398 inscrites comme étranger non admissible au titre de l'article 96. Concernant l'article 96, en 2002, 3027 signalements correspondaient à la découverte sur le territoire national d'un signalement émis par l'un des partenaires Schengen (dont majoritairement l'Allemagne), et 257 signalements à la découverte d'un signalement français sur le territoire d'un partenaire Schengen, soit 3274 découvertes. Si l'on calcule le rendement du SIS sur l'article 96 en exprimant en pourcentage le nombre de découverte d'un signalement français à l'étranger sur le nombre de signalements inscrits ce taux est de $0,49 \%{ }^{47}$. Pour obtenir des données comparatives plus générales, ne sont disponibles que les annexes publiées par l'autorité de contrôle sur les données au 31 Décembre 2001 et au 23 mai $2000^{48}$. Concernant l'année 2001, on avait 10541271 entrées. La France en avait inscrites ou avait répondu à un signalement étranger à hauteur de 3,1 millions, l'Allemagne à 2,7 millions et l'Italie à 2,4 millions sur l'ensemble des items répertoriés entre les articles 95 à 100. Concernant l'année 2000, les entrées sont de 9748 083. La France en avait inscrites ou avait répondu à un signalement étranger à hauteur de 3,1 millions, l'Allemagne à 2,8 millions et l'Italie à 2,2 millions sur l'ensemble des items répertoriés entre les articles 95 à 100. Sur 9,7 millions d'entrées au SIS en 1999, on a les mêmes chiffres pour les trois principaux pays ${ }^{49}$. Mais ces chiffres agrégés sont peu significatifs. Il faut décomposer les données entre signalements émis et réponses à des 
signalements, entre objets et personnes. Or c'est quasiment impossible à partir des statistiques brutes des années 2000 et 2001, ce qui conduit à moins de transparence qu'avant malgré le discours sur les bénéfices de l'intégration de Schengen au sein du traité d'Amsterdam. Mais quand on voit le budget ridicule alloué à l'Autorité de Contrôle c'est un témoignage flagrant du mépris des institutions européennes et du conseil JAI pour la protection des données ${ }^{50}$.

Dès lors les seules données utiles remontent à la période 1997 à 1999. Ainsi, d'après les éléments disponibles, l'Allemagne, parce qu'elle inclut systématiquement les alias des personnes recherchées ${ }^{51}$, et les personnes auxquelles il faut refuser l'entrée sur le territoire, était responsable à elle seule en 1997, 1998, 1999 de près de la moitié des signalements émis dans le SIS sous l'article $96^{52}$.

La France venait en seconde position pour l'année 1997 mais, ensuite l' Italie avait dépassé la France en multipliant les interdictions d'entrée sur le territoire ${ }^{53}$. En revanche, la France est la première à fournir des informations à ses partenaires, ce qui s'explique au vu de sa situation géographique avec peu de frontières extérieures mais beaucoup de frontières intérieures. Elle doit en effet beaucoup travailler pour répondre aux hits des alertes de ses partenaires, ce qui ne simplifie pas la gestion aux frontières.C'est elle qui de très loin fournit les renseignements aux autres sur l'article $96(5756,6392,4952)$, suivie de l'Autriche $(158,2129$ et 2390$)$ et de l'Allemagne (725, 2193, 1650).

Face à cette hyperactivité de l'Allemagne, de la France et de l'Italie, les autres pays ne suivent guère. Ainsi, le Portugal ne déclarait pour les mêmes années quasiment pas de personnes à qui refuser l'entrée $(13,10$ et 8$)$, ce qui présuppose une paix sociale extraordinaire ou plus sûrement une autre conception du SIS. Plusieurs Etats, en particulier les pays nordiques, estiment comme le Portugal, la Grèce ou le Luxembourg que l'inscription au SIS ne devrait se faire que pour des crimes et délits "significatifs » - ce qui vise indirectement la politique allemande d'interdiction systématique du territoire.

Des cours nationales ont aussi été dans ce sens comme le montre Claire Saas dans l'interprétation donnée par le Conseil d'Etat de l'inscription au SIS ${ }^{54}$. Dès lors, l' harmonisation des consultations masque l'extrême disparité des logiques nationales d'inscription des signalements au sein du SIS. Le SIS n'est en aucun cas un reflet cohérent de la criminalité dans les différents pays, même si certains hommes politiques ont voulu s'en servir ainsi. Le fait de maintenir dans le troisième pilier le fonctionnement technique du SIS n'a pas non plus facilité l'harmonisation car aucune instance ne peut vérifier ce que les différents services habilités dans chaque pays entrent dans le SIS en amont de ces entrées. Il n'y a que des possibilités a posteriori de recours. Ceci est particulièrement gênant en ce qui concerne l'usage de l' article 96 dont le contenu touche à l'entrée sur le territoire et dont les individus devraient profiter des standards et recours du droit européen en matière de protection et de droits individuels.

Nous ne disposons pas de données beaucoup plus récentes permettant d'affiner la recherche et de voir comment les pays qui sont entrés après 1998 ont agi. Les seules données disponibles après cette date sont celles publiées par la Haute Autorité de protection des données. Or, celle-ci n'analyse pas les stratégies et ne commente même pas les chiffres. Nous avons signalé dès l' introduction ce paradoxe de la «transparence » d'Amsterdam qui fournit bien moins de données aux chercheurs et à 
la société civile qu'à la période du secrétariat Schengen. Les responsables de la Commission ont justifié l'absence de rapports par le fait que Schengen était maintenant intégré au sein de l'Union; conception paradoxale de la transparence où le fait qu'un rapport - comité Schengen - devrait donner naissance à deux rapports, l'un troisième pilier d'Amsterdam et l'autre premier pilier Titre IV, débouche sur l'absence de rapport intégré et cohérent !

Nous pouvons tout de même utiliser les éléments venant des statistiques de la haute autorité pour 1999 et 2000 et les relier aux tendances du rapport 1998 :

Le SIS est passé de 3868529 hits en 1995, à 4592949 en 1996, et 5592240 signalements en 1997. Il a atteint 8700000 en 1998 (ce qui s'explique par l'entrée des nouveaux membres) et atteignait en 1998 près de 9700000 signalements - chiffre avant l'entrée des pays nordiques - dont 1,3 million sont des signalements de personnes. Parmi ces 1,3 million, 764851 sont des signalements d'indésirables, à comparer avec les 10419 criminels recherchés à fin d'extradition et les 27436 personnes recherchées. Le restant - soit 8,4 millions de signalements - correspond à des objets volés (documents ou véhicules article 100). Le système est consultable depuis plus de 5000 postes frontières, consulats et autres acteurs autorisés. Ces résultats globaux ne font que confirmer ce que nous avions écrit dès 1992, dans notre livre sur l'Europe de la sécurité intérieure, et ensuite dans plusieurs articles, à savoir que le SIS est avant tout un système visant à contrôler les flux migratoires et ne joue pas de rôle déterminant en matière de lutte contre le crime où il ne fait souvent que dupliquer le système Interpol ${ }^{55}$.

Seuls $2 \%$ des personnes inscrites sur le SIS ont commis des crimes graves et uniquement $7 \%$ ont commis des infractions en liaison avec un crime. Les rhétoriques sur son efficacité à l'égard du crime sont donc douteuses. Elles servent de paravent à son usage comme instrument d'une police de l' immigration (89\% des personnes inscrites au SIS le sont sous l'article 96) et renforcent malheureusement la connexion idéologique crime/migration en utilisant, certes sous des rubriques différentes, le même instrument, le SIS, pour la lutte contre le crime et la lutte contre l'immigration. Nous allons donc distinguer ces deux thèmes et essayer de les relier à la question des visas.

Que signifie l'augmentation régulière des chiffres dans le SIS ? Elle ne répond pas à une augmentation du crime ou de l'immigration illégale mais au versement au SIS de données qui étaient souvent stockées ailleurs. Ces données ne sont pas forcément récentes, certains pays ont, pour montrer leur bonne volonté, mis tous les noms ou objets trouvés dans leurs fichiers. Leur pertinence peut être discutée. Pour ne prendre qu'un exemple banal, il est arrivé à quelqu'un d'être arrêté à la frontière en revenant d'un voyage parce qu'on le suspectait de conduire une voiture volée. Investigation faite, c'était un vol qu'il avait déclaré lui-même sept ans auparavant à la police et la voiture avait été retrouvée trois jours après. Les données sont donc stockées et l'accumulation à tout prix est privilégiée souvent sur la pertinence et l'actualité. Le fait que la Haute Autorité en matière de protection des données ait un rôle marginal et n'ait pas de pouvoir de sanction à l'égard des services défaillants est un problème important. La connexion entre SIS et SIRENE est aussi gênante car les bureaux SIRENE sont peu régulés et ils manquent de juges spécialisés dans l'immigration et les libertés publiques. Ces services sont plus le fait de techniciens et parfois leur usage de données plus " sensibles » fournies par les services de renseignements via des services judiciaires qui ne font qu'endosser leurs données, reste un point à surveiller ${ }^{56}$. 
Il est significatif qu'on ne donne aucun chiffre cohérent sur les retraits opérés ces dernières années. Ne retire-t-on aucun signalement ? Un membre de la haute autorité de contrôle se plaignait de ce que leurs recommandations ne soient pas suivies d'effets. Un responsable français critiquait lui plus directement les services italiens pour avoir versé au SIS des signalements anciens d'une dizaine d'années, faits par leurs services de renseignements de l'époque et dont la fiabilité était discutable: tout "gauchiste" ayant été considéré comme subversif est maintenant classé sous la catégorie « indésirable » - personne à surveiller - même si depuis ils ont totalement changé de comportement et sont parfois devenus chefs $d$ ' entreprises. Avec la tentation de réactiver les contrôles aux frontières intérieures lors de sommets comme ceux de l'Union ou du G8, c'est une base incohérente et inefficace d'indésirables qui ressort du SIS.

La question des doubles enregistrements avec les bases Interpol est loin d' être réglée. Cela risque de créer des confusions ou d'affaiblir une base par rapport à l'autre, ce qui obligerait à consulter les deux. Les pays ont des stratégies différentes comme le montre l'exemple portugais où l'on privilégie Interpol.

Si l'on décompose les chiffres par pays et par thèmes, il apparaît que :

Sur l'année 1997, il y a eu 9029 demandes d'informations envoyées vers la France et elle a émis vers le système 3143 demandes. En 1998 les chiffres sont passés à 11139 et 3 074 puis en 1999 à 9485 et 2 899. A contrario, l'Allemagne a reçu, en 1997, 2612 demandes et émis 6625 ; respectivement 4643 et 8507 en 1998 ; et, 3912 et 7726 en 1999. Les deux pays sont de loin ceux qui utilisent le plus le SIS, l'Allemagne par ses demandes, la France par ses réponses. L'Italie est aussi de plus en plus active depuis 1998 ( 2261 reçues et 5060 envoyées) et 1999 (1 284 et 6 507) ainsi que l' Autriche.

Le SIS sert, comme nous l'avons dit, en priorité au refus d'entrée des étrangers en situation illégale : 14806 refus sur la base de l'article 96 en 1997, 23909 en 1998, et 21 711 en 1999 ; ainsi qu'à la recherche de véhicules volés ; 18902 véhicules sur la base de l'article 100 en 1997, 15906 en 1998 et 13917 en 1999.

Néanmoins le SIS permet aussi de signaler des personnes pour des enquêtes criminelles : 3320 personnes recherchées par la justice (arrestation ou témoignages) et 1357 extraditions sont passées via le SIS et le SIRENE en 1997, 2004 demandes d'extraditions en 1998 et 2416 en 1999.

La lutte contre le crime via le SIS est donc marginale dans le volume d' activités du SIS, néanmoins elle représente aussi un plus en termes de lutte. Des personnes recherchées sont retrouvées uniquement grâce au SIS, et ceci parce que la base est plus fréquemment consultée que celle d'Interpol. L'entraide judiciaire a joué pleinement durant les premières années en permettant de retrouver des criminels depuis longtemps installés dans un autre pays européen mais le taux a tendance à diminuer. En revanche, les demandes d'arrestations à fin d'extradition sont en hausse.

Dès lors, le SIS a bien sûr un rôle significatif au sens où, quel que soit le point d'entrée aux frontières extérieures, théoriquement l'agent qui contrôle dispose des mêmes données, qu'il soit policier français, carabinier italien ou douanier autrichien. Mais l'ensemble des données n'en est pas moins disparate selon ce que les pays ont décidé ou non d'entrer. Le nombre impressionnant de données, étant donné la dimension européenne ne doit pas faire oublier ce fait. Les procédures sont harmonisées mais les contenus ne le sont pas. Dès lors, son usage à la frontière comme ultime vérification relève plus de la croyance partagée dans la technologie que de l'efficacité réelle du 
système. Il rassure par les chiffres impressionnants de données conservées à l'intérieur du système mais n'est pas optimalement opérationnel. Il charrie trop d'erreurs, trop d'alias attribués au hasard des enquêtes policières, trop d'entrées de l'Allemagne et de l'Italie et quasiment pas du Portugal ou de la Grèce et de l'Espagne. Le SIS reste ainsi avant tout un moyen technique pour la police des étrangers. Il sert comme nous le verrons plus loin à empêcher des personnes qui étaient entrées illégalement sur le territoire européen et qui en avaient été expulsées, d'y retourner sous la même identité. Par ailleurs, ce n'est pas au hasard que les juridictions nationales qui avaient fait confiance au SIS exigent maintenant beaucoup plus d'éléments et refusent une inscription au SIS comme preuve suffisante ${ }^{57}$.

Pour résumer cette partie, sur les désaccords concernant la politique aux frontières et le rôle de Schengen ainsi que du SIS, on peut dire que les contrôles aux frontières intérieures n'ont pas été complètement abolis mais selon les pays ils ont été plus ou moins assouplis, délocalisés et privatisés. Les contrôles aux frontières extérieures varient selon les politiques gouvernementales des pays ayant une frontière extérieure en fonction du rapport de force avec leur voisin, de l'état de leur opinion publique et de celle des pays voisins, de la possibilité technique de contrôler facilement ou non les frontières, de leurs capacités financières. Les inscriptions au SIS facilitant la collaboration policière varient aussi fortement d'un pays à l'autre, de même que les interprétations juridictionnelles qui en sont faites.

De plus, tout ceci ne concerne que les durées de court séjour et les politiques globales d'immigration ne sont en rien harmonisées ou mêmes convergentes. La tendance la plus forte à la convergence tient au poids politique que l'Allemagne et la France entendent donner à cette question et à l'arrivée récente du Royaume-Uni dans ces logiques. Les autres pays ont tendance à plier devant les exigences majeures de ces Etats, de même que la Commission lorsqu'elle prépare ses réflexions. Les résistances sont souvent à la marge et ne renvoient que très rarement à un affrontement ouvert sauf en cas de désaccord majeur entre la France et l'Allemagne. A l'intérieur des deux pays, ce sont les ministères de l'Intérieur et de la Justice qui ont le poids le plus considérable dans ces questions si l'on compare leur influence avec celle des ministères de l'Industrie ou aux Affaires étrangères.

Mais jusqu'où ces ministères partagent-ils les croyances des premiers? $C^{\prime}$ est au Royaume-Uni que l'on a vu une attaque en règle du dogme de la maitrise de l'immigration et le renversement du discours en faveur d'une immigration qualifiée pouvant combler le déficit d'emploi résultant du déficit démographique. La Commission a aussi très rapidement essayé $d$ ' exploiter ce contre-discours. Les ministères des Affaires étrangères ont un rôle de plus en plus fort avec les procédures d'Amsterdam. Ils sont moins focalisés sur la frontière et, au moins en Allemagne, ont la volonté de jouer un rôle via la politique commune des visas.

Ceci nous amène au cour de notre recherche. La politique commune des visas est-elle une stratégie alternative de police à distance visant à remédier aux difficultés de la gestion aux frontières ou n'en est-elle qu'un point technique ? Est-elle centrale car peu visible ou marginale dans une économie globale de contrôle des frontières? Nous estimons qu'elle est devenue un point central de l'économie du dispositif de mise à l'écart des étrangers ou ban-optique qui amène à contrôler de plus en plus certains individus déterminés à travers des profils de risque et qu'elle renforce nombre d' injustices à l'échelle globale, et ce, d'autant plus que peu visible et peu discutée, 
considérée comme technique, elle échappe au regard des citoyens et des ONG les plus concernées par ce procédé même de la gouvernementalité à distance.

\section{NOTES}

1. . Bigo Didier, « Vers une Europe des polices? », Revue Française d' Administration Publique ${ }^{\circ} 91$, juillet-septembre 1999, pp. 471-482 sur le fait que les Européens croient et ne croient pas, comme les Grecs de Paul Veyne, à leurs mythes. Voir aussi Sciortino Giuseppe et Ferrucio Pastore, Immigration and european immigration policy : myth and realities, paper, European academy, Triere, July 2002.

2. . Sur les raisons qui ont poussé à l'accord de Schengen de 1985, grève des douaniers italiens, grève réactive des camionneurs et paralysie du territoire français, protestation des autres gouvernements d'une part, stratégie bureaucratique du secrétariat du Benelux d'autre part, voir Bigo Didier, Polices en réseaux, op. cit.

3. . Pour une discussion plus approfondie de l'intergouvernementalité dans Schengen et le troisième pilier, voir Pauly Alexis, « De Schengen à Maastricht : voie royale et course d'obstacles », De Schengen à Maastricht, EIPA Maastricht, 1996 ; den Boer Monica, « The Implementation of Schengen : First the Widening, Now the Deepening ", De Schengen à Maastricht, EIPA Maastricht, 1997 ; d 'Oliveira J., « Expanding External and Shrinking Internal Borders : Europe's Defence Mechanisms in the Area of Free Movement, Immigration and Asylum ", in D. O'Keeffe and P. Twomey, Legal Issues of the Maastricht Treaty, Chancery Law Publishing, Chichester, 1994.

4. . Sur l'attitude du gouvernement britannique : si celui-ci refusait d'interpréter le futur Acte Unique Européen en discussion comme une contrainte et s'il tenait à conserver le contrôle de ses frontières, il était en revanche celui qui poussait le plus à la collaboration policière. Voir son rôle dans le police working group, dans Trevi et dans la préparation de l'UDE Europol.

5. . Entretien avec un des premiers négociateurs de l'accord de Schengen de 1985. Entretien de 1991.

6. . On sait à quel point la ratification des accords intergouvernementaux de ce type a été un échec en termes de souplesse et de rapidité. C'est d'ailleurs pourquoi on a institué à Maastricht une autre solution, celle des piliers.

7. . Ces dispositions ont été remplacées par la Convention relative à la détermination de l'Etat responsable de l'examen de la demande d'asile (Convention de Dublin) du 14 juin 1990, lorsque celle-ci est entrée en vigueur le 1er septembre 1997.

8. . Albrecht Funk, « Les mythes du contrôle : la frontière orientale de la République Fédérale d'Allemagne au tournant des années 1990 », Cultures \& Conflits, n²4/25, Paris, L' Harmattan, 1997.

9. . Sur le SIS et ses implications voir Mathiesen Thomas, « On Globalisation of Control : Towards an Integrated Surveillance System in Europe », Statewatch, 2000.

10. . Sur les CCPD voir Maguer Azilis : « La coopération policière transfrontalière », Cultures \& Conflits, Approches Comparées des Polices en Europe, $n^{\circ} 48$, Paris, L'Harmattan, Hiver 2002. 
11. . Par ailleurs la France, seule, fait jouer la clause de sauvegarde pour maintenir des contrôles aux frontières de la Belgique et du Luxembourg en arguant du petit trafic transfrontalier de drogue tenant à la législation des Pays-Bas, alors que l'Allemagne ne le fait pas. Elle a aussi durant les séries d'attentats de 1995-96 renforcé à tel point ces contrôles mobiles et aléatoires qu'ils revenaient en pratique à des contrôles systématiques, mais n'a pas pour autant fait jouer la clause de sauvegarde à toutes ses frontières.

12. . L'Italie a signé les accords le 27 novembre 1990, l'Espagne et le Portugal, le 25 juin 1991, la Grèce, le 6 novembre 1992, l'Autriche, le 28 avril 1995 ainsi que le Danemark, la Finlande et la Suède, le 19 décembre 1996.

13. . Voir également, House of Lords, European Communities - 31st Report, Session 1997-98, Incorporating the Schengen acquis into the European Union, London 1998.

14. . Bigo Didier, « Les non-dits de la sécurité intérieure » in Lequesne Christian, De La Serre Françoise, Quelle Union pour quelle Europe ?, Complexe, mai 1998.

15. . Rossetto J., « Le contrôle judiciaire dans la Convention de Schengen et dans le troisième pilier ", in den Boer M., op. cit., pp. 27-40 ; Boeles P., Fair Immigration Proceedings in Europe, Kluwer Law International, La Haye, 1997, Chapitre 16, pp. 361-366 ; Groenendijk K., " The European Court of Justice and the Third Pillar », in Meijers H. et al., Democracy, Migrants and Police in the European Union : the 1996 IGC and Beyond, Forum, Utrecht, 1997, pp. 45-61.

16. . « Le gouvernement [de Grande-Bretagne] a hérité de ses prédécesseurs une politique consistant à se garder une porte de sortie (opting out) et à privilégier le troisième pilier comme étant un moyen supérieur de la coopération entre Etats membres, avec le minimum d' interférences de la part de la Commission, du Parlement européen ou de la Cour de Justice des Communautés Européennes ». House of Lords, European Communities, op. cit., paragraphe 81.

17. Guild Elspeth, Immigration Law in the European Community, op. cit.

18. . Voir la communication de la Commission au Conseil et au parlement Européen : vers une gestion intégrée des frontières extérieures des Etats-membres de l'UE Com (2002) 233 final du 07/05/2002 et a contrario le document de la présidence espagnole du 30/05/2002 corps de garde-frontières européen : histoire et étude de viabilité réalisée par l'équipe de projet italienne.

19. . Voir les travaux de Cruz Antonio, Carrier Liability in the Member States of the European Union, Brussels : CCME Briefing Paper, (17 september), 1995 et ses comptesrendus réguliers sur la question dans la migration news letter. Voir Guiraudon Virginie, op. cit. Voir également Weber Frank Paul, Le contrôle par la Lufthansa des passagers, contrôler en amont ?, paper ISA Toronto 1996, 10 pages ; « Expulsion : genèse et pratique d'un contrôle en Allemagne ", Cultures \& Conflits, Circuler, enfermer, éloigner, $n^{\circ} 23$, automne 1996.

20. . Initiative de la République française en vue de l'adoption de la directive du Conseil relative à l'harmonisation des sanctions pécuniaires imposées aux transporteurs acheminant sur le territoire des États membres des ressortissants des pays tiers démunis des documents nécessaires pour y être admis. JO C 269 (2000), pp. 0008 - 0009. 21. JOCE L187/45, 2001. Pour une analyse précise des sanctions aux entrepreneurs, voir Antonio Cruz, op. cit., Guild E., « Immigration law from a migrant perspective », Nijmegen, 1999, et Guiraudon Virginie, « Before the EU Border : Remote Control of the "Huddled Masses" » op. cit. 
22. Wiener Antje, European citizenship practice, building institutions of a non state, Boulder, Westview Press, 1998 ; Koslowski Rey, « Personal Security, State Sovereignty and the Deepening and Widening of European Cooperation in Justice and Home Affairs » in Guiraudon Virginie et Christian Joppke (eds), Controlling a New Migration World, London, Routledge, 2001.

23. . On notera malgré tout la capacité du Royaume-Uni d' avoir pu seul - avec l'Irlande - avoir le choix d'un Schengen à la carte, choix refusé à tous les autres pays par les membres du noyau initial qui le refusèrent même à l'Italie alors que la Convention n'était pas appliquée. La notion d'acquis mériterait de nombreux commentaires mais cela s'éloigne trop de notre sujet.

24. . Bigo Didier, « Le champ européen de l' (in)sécurité » in Fortmann Michel, Stéphane Roussel et Alex Macleod (éd.), Vers des périmètres de sécurité ? La gestion des espaces continentaux en Amérique du Nord et en Europe, Montreal, Athéna éditions, 2003.

25. . Voir Lavenex Sandra, «Transgressing borders : The emergent European refugee regime and safe third countries ", in Peters P. et Cafruny A. (eds.), The Union and the World, The Hague, Kluwer Law International, 1998, pp.113-132 ; « Passing the Buck' : European Union refugee policies towards Central and Eastern Europe », Journal of Refugee Studies, 1998, 11(2), pp. 126-145 ; « Asylum, Immigration and Central-Eastern Europe : Challenges to EU Enlargement », European Foreign Affairs Review, 1998, 3 (2), pp. 275-294 ; Safe Third Countries. Extending EU Asylum and Immigration Policies to Central and Eastern Europe, Budapest, Central European University Press, 1999.

26. . Voir l'annexe sur la composition de la DG JAI (Cultures \& Conflits, n50, Eté 2003). 27. . La coopération judiciaire en matière civile a également été introduite dans le nouveau titre du Traité CE. Voir « De Tampere à Séville : bilan de la sécurité européenne », Cultures \& Conflits, $n^{\circ} 45$ et n 46 , Paris, L'Harmattan, 2002.

28. . JO L 119, 7.05.1999.

29. . JO L 176, 10.07.1999 - rectificatif : Journal officiel L 9, 13.01.2000.

30. . Voir supra.

31. . JO C 19 du 23.1.1999.

32. . Règlement (Conseil d'Etat) $\mathrm{N}^{\circ} 539 / 2001$ du Conseil du 15 mars 2001 fixant la liste des pays tiers dont les ressortissants sont soumis à l' obligation de visa pour franchir les frontières extérieures des Etats membres et la liste de ceux dont les ressortissants sont exemptés de cette obligation. JOCE du 21 mars 2001, L81/1 (Cultures \& Conflits, $n^{\circ} 50$, Eté 2003).

33. . Proposition de règlement de la Commission n 5392001.

34. . Ces deux listes ont servi à constituer la carte présentée dans ce numéro. Voir également Cultures \& Conflits, $n^{\circ} 50$, Eté 2003)..

35. . [1] JO L 234 du 3.10.1995, p. 1.

36. . [2] JO L 72 du 18.3.1999, p. 2.

37. . Voir, pour plus de détails, l' article de Gérard Beaudu qui actualise les données. L'Espagne, dont les liens avec ce pays sont les plus forts, a justifié cette inscription auprès des autorités colombiennes par l'impossibilité de déroger à une décision communautaire. On voit l'avantage que les gouvernements voient maintenant dans le règlement. Il est bien plus simple de se retrancher derrière une position commune que de décider clairement de soumettre les ressortissants colombiens à visa.

38. . Les relations avec l'Islande et la Norvège : ces deux pays appartiennent avec la Suède, la Finlande et le Danemark à l'Union nordique des passeports qui a supprimé les 
contrôles à leurs frontières communes. La Suède, la Finlande et le Danemark devenus signataires des accords de Schengen grâce à leur statut d'États membres de l'UE, l'Islande et la Norvège ont été associés à leur développement depuis le 19 décembre 1996. Sans disposer d'un droit de vote au sein du Comité exécutif de Schengen, ils avaient la possibilité d'exprimer des avis et formuler des propositions. Pour prolonger cette association, un accord a été signé le 18 mai 1999 entre l'Islande, la Norvège et l'UE [JO L 176, 10.7.1999]. Ces pays continuent à participer à l'élaboration de nouveaux instruments juridiques ayant trait au développement de l'acquis de Schengen. Ces actes sont adoptés uniquement par les États membres de l'UE mais s'appliquent également aux pays précités. Dans la pratique, cette association prend place sous la forme d'un Comité mixte, créé en dehors du cadre de l'UE. Il réunit des représentants des gouvernements d'Islande et de Norvège, des membres du Conseil de l'UE et de la Commission. Des procédures ont été définies pour la notification et l'acceptation de futures mesures ou actes. Le Comité a adopté son règlement intérieur le 29 juin 1999 [JO C 211, 23.7.1999]. Dans les domaines de l'acquis de Schengen qui s'appliquent à l'Islande et à la Norvège, les relations entre ces deux pays, d'une part, et l'Irlande et le RoyaumeUni d'autre part, sont réglées par un accord approuvé par le Conseil le 28 juin 1999 [Journal officiel L 15, 20.01.2000].

39. . Décision $n^{\circ}$ 91-294 du Conseil Constitutionnel sur la loi autorisant l'approbation de la convention d'application de l' accord de Schengen du 14 juin 1985.

40. . Wenceslaw de Lobkowicz, L'Europe de la sécurité intérieure, Paris, La Documentation Française, 2002.

41. . Conseil schengen4 7994/00 et schengen5 7995/00 du 20/04/2000 et du 26/04/2000 demande du Royaume-Uni de participer à certaines dispositions de l'acquis Schengen. 42. . Le C-SIS n'est pas une base supplémentaire ou même un fichier de traitement, $\mathrm{C}^{\prime}$ est un index de signalement renvoyant aux fichiers nationaux. La structure acéphale du SIS diffère de celle des fichiers Europol.

43. . Le SIRENE France relève de la Direction Centrale de la Police Judiciaire mais le bureau qui s'en occupe associe la gendarmerie. Il existe une mission Justice auprès de la DCPJ qui est placée sous l'autorité d'un magistrat de l'administration centrale du ministère de la Justice. Sur ces éléments voir les travaux de Daniel Fontanaud qui fut le premier magistrat Schengen dont : " La coopération judiciaire en Europe », Problèmes politiques et sociaux, $\mathrm{n}^{\circ}$ 786, La Documentation française, 1997, $80 \mathrm{p}$.

44. . JO L 123, 13.5.1999.

45. . Décision du Conseil du 6 décembre 2001 relative au développement du système information Schengen de deuxième génération (SIS II), Journal officiel des Communautés européennes du 13 décembre 2001, L. 328/1.

46. . Une thèse soutenue à l' université de Nijmegen par Evelin Brouwer est en train d'être terminée sur ce sujet particulièrement important.

47. . Pour l'analyse de ces taux et une présentation qui rompt heureusement avec le discours d'auto-louanges habituelles concernant le SIS, voir la communication de Jean Yves Lesaffre au colloque sur l'effectivité de la sanction pénale à propos des origines de Schengen et du rôle du SIS.

48. . Joint Supervisory Authority Schengen 5th Report Brussels (pas de date de publication).

49. . Joint Supervisory Authority Schengen 4th Report Brussels, 18 Juillet 2000, SCHAC 2533/100 rev1.

50. . Le budget total en 1999 était de 137580 euros soit 0,021\% du budget Schengen. 
51. . Près de 470000 alias en 99 dans le système dont plus de $90 \%$ ont été entrés par l'Allemagne!

52. . Respectivement 3999, 5273, et 4275 hits venant de ses partenaires à ses alertes.

53. . 443, 1121, 2781 .

54. . Voir son article dans le second volume de ce numéro.

55. . Bigo Didier (dir.), L'europe des polices et de la sécurité intérieure, op. cit.

56. . Sur ces différents points et pour une approche théorique voir Mathiesen Thomas, Siste Ord Er Ikke Sagt Schengen Og Globaliseringen Av Kontroll, Pax Fortag, Oslo, 2000 et le résumé en anglais dans Statewatch, « On Globalisation of Control : Towards an Integrated Surveillance System in Europe », op.cit.

57. . Nous verrons ce point dans l'article de Claire Saas, déjà cité, qui aborde la manière dont les Cours françaises interprètent la validité d'une inscription au SIS, mais ceci est vrai aussi en Belgique, aux Pays-Bas et en Italie.

INDEX

Mots-clés : frontières, Visa, construction européenne, police à distance

Index géographique : Schengen, Union européenne 